\title{
Modulation of Host Autophagy during Bacterial Infection: Sabotaging Host Munitions for Pathogen Nutrition
}

\author{
Pedro Escoll ${ }^{1,2}$, Monica Rolando ${ }^{1,2}$ and Carmen Buchrieser ${ }^{1,2 *}$ \\ ${ }^{1}$ Institut Pasteur, Biologie des Bactéries Intracellulaires, Paris, France, ${ }^{2}$ CNRS UMR 3525, Paris, France
}

Keywords: autophagosome, xenophagy, autophagy modulators, intracellular bacteria, bacterial nutrition

\section{AUTOPHAGY IS A DEFENSE MECHANISM AGAINST INVADING PATHOGENS}

OPEN ACCESS

Edited by:

Abhay Satoskar,

The Ohio State University, USA

Reviewed by:

Eric Ghigo,

Centre national de la recherche

scientifique, France

Robert Heinzen,

National Institutes of Health, USA

Soubeyran Philippe,

Institut national de la santé et de la

recherche médicale, France

*Correspondence:

Carmen Buchrieser cbuch@pasteur.fr

Specialty section: This article was submitted to Microbial Immunology, a section of the journal

Frontiers in Immunology

Received: 21 December 2015 Accepted: 19 February 2016

Published: 03 March 2016

Citation:

Escoll P, Rolando M and Buchrieser C (2016) Modulation of

Host Autophagy during Bacterial Infection: Sabotaging Host Munitions for Pathogen Nutrition. Front. Immunol. 7:81. doi: 10.3389/fimmu.2016.00081
Cellular homeostasis requires the balanced regulation of anabolic and catabolic processes. While anabolic metabolism consumes energy to build up cellular components, catabolic processes break down organic matters in order to provide energy for the cell and its anabolic processes. Autophagy is a highly conserved and regulated catabolic process by which the eukaryotic cell degrades unnecessary, undesirable, or dysfunctional cellular components, including organelles (1-3). Autophagy is induced by a variety of extra- and intracellular stress stimuli, such as nutrient starvation, oxidative stress, or accumulation of damaged organelles or toxic protein aggregates. Initiation of autophagy first leads to the formation of cup-shaped structures known as phagophores that engulf the undesirable or damaged cellular components. Subsequent elongation of phagophores form double-membrane vesicles called autophagosomes, which deliver their cargo to lysosomes where the content is degraded and recycled (1-3). Autophagy plays a central role in quality control of organelles and proteins, and additionally is a key mechanism to maintain cellular energy levels and nutrient homeostasis during starvation, promoting the recycling and salvage of cellular nutrients. Furthermore, the cellular autophagic machinery is also used to remove invading intracellular pathogens, a process called xenophagy $(1,2)$. In this case, phagophores engulf invading microbes forming autophagosomes and steering them toward lysosomal degradation. Thus, xenophagy is an innate immune mechanism against bacterial infection that has been shown to be essential to restrict intracellular growth of many bacteria such as Salmonella enterica serovar Typhimurium (4), Mycobacterium tuberculosis $(5,6)$, Listeria monocytogenes (7), or Group A Streptococcus (8).

Detection of bacterial components in the cytoplasm of mammalian cells induces autophagy via the activation of toll-like receptor 4 (TLR4) by bacterial lipopolysaccharide (LPS) and recognition of bacterial peptidoglycan by NOD1 and NOD2 $(9,10)$. TLR- and NOD-like receptor (NLR)-induced autophagy can be initiated during entry, uptake, or phagocytosis of bacteria by the host cell $(10,11)$, but bacteria can also be sensed by the Sequestosome-1-like receptors (SLRs) when they are already in the cytosol (1) (Figure 1A). In both cases, recruitment of autophagy proteins to the phagosome, such as the ULK1 complex, Beclin1, and ATG16L1, initiates membrane nucleation of the phagophore that will engulf the intracellular bacteria (10-12) (Figure 1B). ATG5-ATG12 associates with ATG16L1 and the ATG5-ATG12-ATG16L1 complex facilitates the addition of a phosphatidylethanolamine (PE) group to the carboxyl terminus of LC3, which function together with other factors to assemble, elongate, and allow the closure of nascent autophagosomes (1) (Figure 1C). In addition to this canonical mechanism of autophagy, phagosomes containing bacteria can recruit directly LC3, a process called LC3-associated phagocytosis (LAP). Upon delivery to phagosomes, LC3 promotes phagosome 


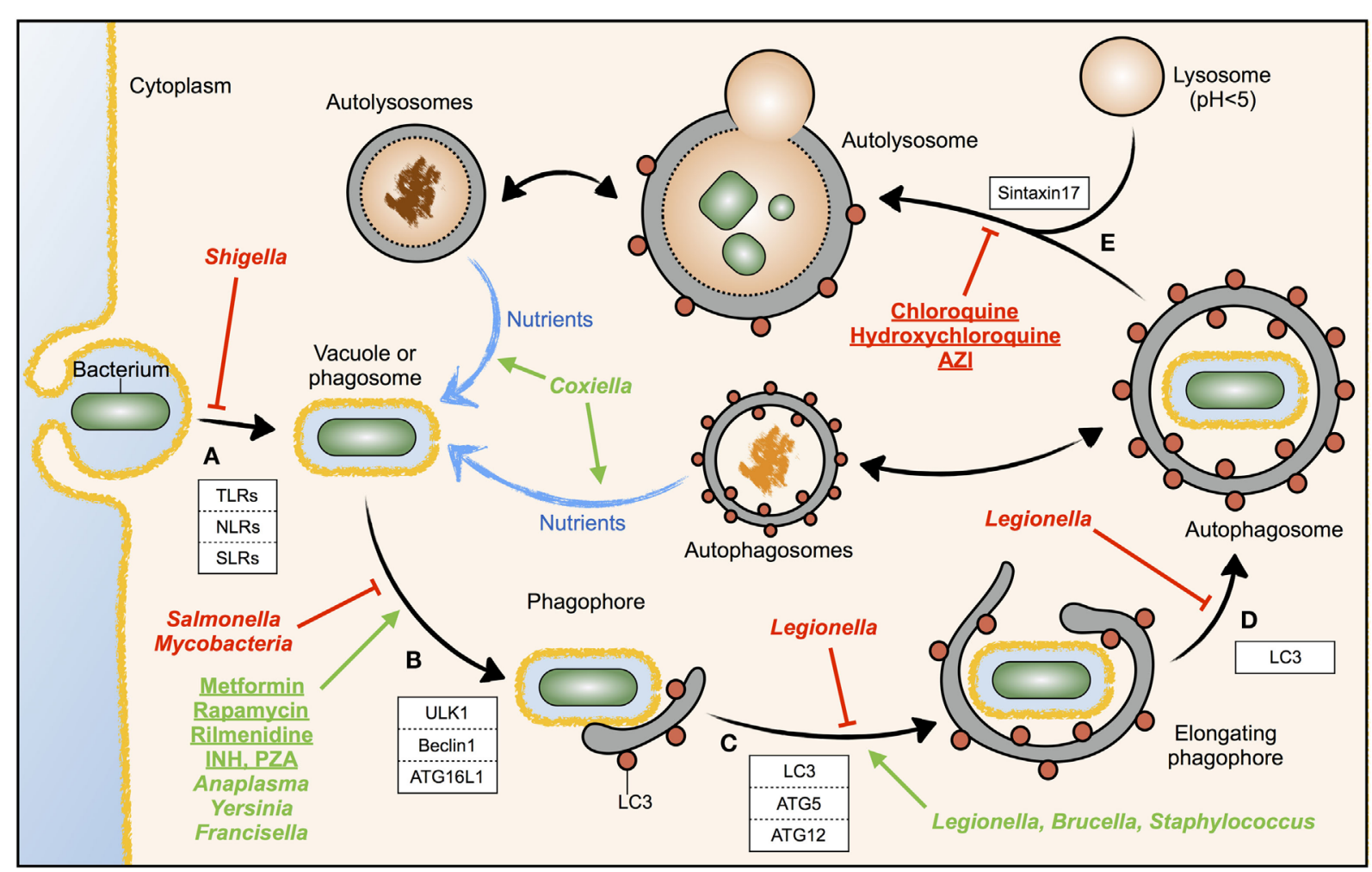

FIGURE 1 | Modulation of autophagy by drugs or intracellular bacteria. The different steps of the autophagic response during bacterial invasion are shown. The host factors known to participate in each step are depicted in white boxes. (A) Invading bacteria are sensed by immune receptors; (B) vesicle nucleation induced by specialized autophagy proteins; (C) phagophore elongation; (D) autophagophore completion; (E) autophagosome maturation by fusion with lysosomes, forming autolysosomes. Drugs (underlined) or intracellular bacteria (cursiva) inducing autophagy are displayed in green, while those inhibiting autophagy are displayed in red. The different steps where bacteria or drugs act are pointed with green arrows (activation) or red T-bars (inhibition). Blue arrows indicate nutrient flow, while doubled-headed arrows indicate the possibility that the content of cellular autophagosomes and autolysosomes can be diverted to the phagosome and used by pathogenic bacteria as a source of nutrients.

maturation and degradation of the content. Therefore, both LAP and canonical autophagy involve the enclosure of bacteria in an LC3-decorated compartment that is targeted for degradation by fusion with the lysosome (2). Membranes from the ER, the Golgi apparatus, the ER-mitochondria contact sites, or the plasma membrane contribute to the elongation of the double membrane of the phagophore in order to form the autophagosome (1) (Figure 1D). The attachment of syntaxin 17 to the autophagosomal membrane enables the fusion with lysosomes and represents the final maturation step of autophagosomes into autolysosomes (13) (Figure 1E), which normally leads to bacterial degradation in case of infection-induced autophagy (2).

\section{PATHOGENIC INTRACELLULAR BACTERIA SUBVERT AND EXPLOIT THE AUTOPHAGY MACHINERY OF THE HOST}

Xenophagy is a defense mechanism of the infected cell against invading bacteria, but intracellular pathogens have evolved mechanisms to inhibit or modulate the autophagy response of the host. For example, M. tuberculosis and Salmonella Typhimurium inhibit autophagy initiation signaling upstream autophagosome formation $(14,15)$, whereas Shigella flexneri evades autophagy recognition by masking the bacterial surface (16) (Figure 1, cursive, red).

In contrast to inhibition of autophagy, certain pathogenic intracellular bacteria induce autophagy and take advantage of it (17) (Figure 1, cursive, green). These bacteria show defective replication in autophagy-deficient cells, and treatment of host cells with autophagy activators promotes bacterial replication. This observation raises the question, why a pathogen would increase a host defense mechanism like autophagy? In uninfected cells, augmentation of the autophagy rate is used to increase the intracellular pool of basic nutrients, to build new cellular structures. During infection, some intracellular bacteria have developed mechanisms to hijack the autophagosomes and redirect the byproducts of the autophagic degradation toward microbial replication rather than for the use by the host cell (18). In most cases, these bacteria actively induce autophagy but, at the same time, block autophagosome maturation and fusion with the lysosome. In this case, augmentation of autophagy, rather than promoting bacterial clearance, promotes the acquisition of nutrients by the invading bacteria (18). Thus, certain bacteria may sabotage the 
host defense mechanism elicited by autophagosomes to use the autophagic vesicles as nutrient source for microbial growth.

An example is Anaplasma phagocytophilum that uses a secreted effector, Ats-1, to promote autophagosome nucleation and stimulates its own growth by using the nutrients contained in the autophagosomes (19). Indeed, autophagy induction using rapamycin favors bacterial infection, while autophagy inhibition decreases A. phagocytophilum replication (20). Another example is Yersinia pseudotuberculosis, a Gram-negative bacterium that replicates intracellularly by establishing a specialized compartment, the Yersinia-containing vacuole (YCVs), which accumulates autophagy markers (21). The stimulation of autophagy with rapamycin increases the size of the YCVs and the numbers of replicative bacteria in the YCVs, whereas autophagy inhibition restricts bacterial survival, suggesting that autophagy promotes Y. pseudotuberculosis replication (21). Yersinia pestis also replicates within YCVs decorated with autophagosome markers (22). The authors suggested that autophagosomes may provide a source of membrane, along with late endosomes, for the expansion of the YCV into a spacious compartment (22). The same mechanism was described for Coxiella burnetii, the causative agent of $\mathrm{Q}$ fever. Coxiella-replicative vacuoles (CRVs) are decorated with the autophagy proteins LC3, Beclin1, and Rab24, and overexpression of LC3 or Beclin1 increases the number and size of the CRVs $(23,24)$. Similar to A. phagocytophilum and Y. pseudotuberculosis, autophagy induction increases $C$. burnetii replication, while inhibition of autophagy blocks Coxiella vacuole formation (23, 25). Also, Francisella tularensis, a highly virulent Gram-negative bacterium responsible for tularemia, avoids xenophagy while inducing autophagy (26). It was shown that autophagy-derived radiolabeled amino acids are transferred from host proteins to F. tularensis, a process that was reduced when host cells were treated with autophagy inhibitors (26).

Other bacteria also co-opt the autophagic machinery for their benefit, although a direct relationship of host autophagy and pathogen nutrition has not been shown. Brucella abortus that causes brucellosis in humans replicates in ER-derived Brucella-containing vacuoles (BCVs). BCVs hijack autophagosome initiation factors, such as ULK1 or Beclin1, and become autophagosome-like compartments (27). Depletion of ULK1 and Beclin1, as well as pharmacological inhibition of autophagy, readily reduced BCV formation, suggesting that autophagy promotes B. abortus infection (27). Staphylococcus aureus was also reported to be sequestered in LC3-positive autophagosomes that evade the fusion with lysosomes (28). S. aureus uses $\alpha$-toxin to induce autophagy by an ATG5-dependent mechanism that also involves reduction of cellular cAMP levels (29). Infection of cells depleted of ATG5 show decreased bacterial replication, showing that autophagy is necessary for $S$. aureus replication in vitro (28).

Thus, different pathogenic bacteria seem to employ a common strategy to subvert the autophagy machinery as they not only target autophagy proteins to block xenophagy set up by the cell to resist infection but also exploit autophagy to promote their own replication. One well studied example for this dual strategy is the Gram-negative intracellular bacterium Legionella pneumophila. After phagocytosis, the causative agent of Legionnaires' disease, forms a Legionella-containing vacuole (LCV) that recruits vesicles emerging from the endoplasmic reticulum (ER) and acquires autophagy markers like LC3, showing that LCVs rapidly become autophagosomes $(30,31)$. This process seems to be dependent on the T4SS bacterial effector LegA9, which promotes the recognition of the LCV by autophagy (32). Interestingly, inhibition of autophagy in permissive $\mathrm{A} / \mathrm{J}$ mouse macrophages reduces Legionella survival at $2 \mathrm{~h}$ postinfection $(30,33)$, suggesting that routing the LCV to the autophagy pathway is beneficial for the bacteria. However, later, it has been shown that L. pneumophila is also restraining autophagy by secreting the specialized effectors, LpSPL and RavZ, that inhibit, autophagosome formation and maturation, respectively $(34,35)$. The paradoxical existence in Legionella of bacterial effectors having opposite roles, on one hand, targeting the LCV to autophagy and, on the other hand, inhibiting autophagy may reflect the necessity for the bacteria to fine-tune host autophagy in a very balanced way. Legionella may need to target the LCV to autophagosomes, avoiding immediate killing (33), and at the same time, it needs to delay the maturation of the LCV-containing autophagosome into autolysosomes, gaining precious time for pathogen replication $(30,36)$.

\section{AUTOPHAGY MODULATORS IN INFECTIOUS AND NON-INFECTIOUS DISEASES: SOME CONSIDERATIONS}

Autophagy modulators are of great interest for medical purposes (37), as it was suggested that metabolic, neurodegenerative, infectious, and oncology diseases can benefit from autophagy modulation (3).

One can hypothesize that drugs inducing autophagy could increase bacterial clearance in infected cells. This hypothesis is supported by the fact that antibiotics widely and extensively used against the intracellular bacterium M. tuberculosis, isoniazid (INH), and pyrazinamide (PZA), although able to kill the bacteria directly in vitro $(38,39)$, have been recently shown to induce autophagy in the host cell promoting mycobacterial clearance (40). Moreover, autophagy is required for effective antimycobacterial drug action in vivo, suggesting that pharmacological modulation of autophagy could be a successful strategy against infections by intracellular bacteria $(40,41)$. This point of view was corroborated by another recent report showing that treatment of cystic fibrosis patients with the antibiotic azithromycin (AZI) was associated with opportunistic mycobacterial infections. AZI was shown to prevent lysosomal acidification and thereby impaired autophagic degradation of mycobacteria (42), suggesting that chronic use of the drug may predispose to mycobacterial disease. Thus, these reports suggest that induction of autophagy with drugs, such as INH or PZA, could successfully treat mycobacterial infections, while inhibition of autophagy with drugs, such as AZI, may in turn facilitate mycobacterial infections.

Similar to mycobacteria, several molecules inducing autophagy have been recently shown to reduce Salmonella Typhimurium replication in HeLa cells $(43,44)$. This direct relationship between drugs, modulating autophagy and the outcome of bacterial infection, emphasizes the essential role of autophagy 
in the host response to intracellular bacteria and seems to support pharmacological modulation of host autophagy during infection. Unfortunately, as shown above, the situation seems more complex than the conclusion "increase of cellular autophagy favors bacterial clearance."

The fact that autophagy inducers seem to be helpful in the treatment of Mycobacteria or Salmonella infections, but in turn might facilitate infections by Anaplasma, Coxiella, Yersinia, or Francisella, requires not only to be highly cautious in the use of autophagy modulators to treat infectious diseases but also to monitor the infectious risk during the use of autophagy modulators. Some autophagy modulators are already in use (Figure 1, underlined). Rapamycin, metformin, and rilmenidine, all autophagy inducers, are drugs approved and prescribed to prevent rejection of kidney transplants, to treat type 2 diabetes, and to treat hypertension, respectively $(3,37)$. In contrast, chloroquine and hydroxychloroquine, which are now under clinical trials as autophagy inhibitors for the treatment of certain resistant cancers, are drugs prescribed to treat malaria $(3,37)$. Moreover, hydroxychloroquine combined with doxycycline is currently used to treat Coxiella-induced chronic Q fever endocarditis (45). Some of these approved drugs might thus show a therapeutical benefit in case of infection.

\section{REFERENCES}

1. Deretic V, Saitoh T, Akira S. Autophagy in infection, inflammation and immunity. Nat Rev Immunol (2013) 13:722-37. doi:10.1038/nri3532

2. Huang J, Brumell JH. Bacteria-autophagy interplay: a battle for survival. Nat Rev Microbiol (2014) 12:101-14. doi:10.1038/nrmicro3160

3. Rubinsztein DC, Codogno P, Levine B. Autophagy modulation as a potential therapeutic target for diverse diseases. Nat Rev Drug Discov (2012) 11:709-30. doi: $10.1038 / \mathrm{nrd} 3802$

4. Birmingham CL, Smith AC, Bakowski MA, Yoshimori T, Brumell JH. Autophagy controls Salmonella infection in response to damage to the Salmonella-containing vacuole. JBiol Chem (2006) 281:11374-83. doi:10.1074/jbc.M509157200

5. Gutierrez MG, Master SS, Singh SB, Taylor GA, Colombo MI, Deretic V. Autophagy is a defense mechanism inhibiting BCG and Mycobacterium tuberculosis survival in infected macrophages. Cell (2004) 119:753-66. doi:10.1016/j.cell.2004.11.038

6. Zhao Z, Fux B, Goodwin M, Dunay IR, Strong D, Miller BC, et al. Autophagosome-independent essential function for the autophagy protein Atg5 in cellular immunity to intracellular pathogens. Cell Host Microbe (2008) 4:458-69. doi:10.1016/j.chom.2008.10.003

7. Py BF, Lipinski MM, Yuan J. Autophagy limits Listeria monocytogenes intracellular growth in the early phase of primary infection. Autophagy (2007) 3:117-25. doi:10.4161/auto.3618

8. Nakagawa I, Amano A, Mizushima N, Yamamoto A, Yamaguchi H, Kamimoto T, et al. Autophagy defends cells against invading Group A Streptococcus. Science (2004) 306:1037-40. doi:10.1126/science.1103966

9. Xu Y, Jagannath C, Liu X-D, Sharafkhaneh A, Kolodziejska KE, Eissa NT. Toll-like receptor 4 is a sensor for autophagy associated with innate immunity. Immunity (2007) 27:135-44. doi:10.1016/j.immuni.2007.05.022

10. Travassos LH, Carneiro LAM, Ramjeet M, Hussey S, Kim YG, Magalhães JG, et al. Nod1 and Nod2 direct autophagy by recruiting ATG16L1 to the plasma membrane at the site of bacterial entry. Nat Immunol (2010) 11:55-62. doi:10.1038/ni.1823

11. Sanjuan MA, Dillon CP, Tait SWG, Moshiach S, Dorsey F, Connell S, et al. Toll-like receptor signalling in macrophages links the autophagy pathway to phagocytosis. Nature (2007) 450:1253-7. doi:10.1038/nature06421

12. Kageyama S, Omori H, Saitoh T, Sone T, Guan J-L, Akira S, et al. The LC3 recruitment mechanism is separate from Atg9L1-dependent membrane
In summary, the study of autophagy regulation during bacterial infection certainly shows the existence of a critical balance between a host-protective "immune-related" induction of autophagy (xenophagy) and a host-deleterious "metabolicrelated" induction of autophagy by invading bacteria for nutritional theft of host energy resources. Results of clinical trials using autophagy modulators and a more profound understanding of the role of autophagy during infection are thus needed to correctly use autophagy modulators in the fight against infectious diseases.

\section{AUTHOR CONTRIBUTIONS}

All authors listed, have made substantial, direct and intellectual contribution to the work, and approved it for publication.

\section{FUNDING}

Work in CB's laboratory is financed by the Institut Pasteur, the Institut Carnot-Pasteur MI, the French Region Ile de France (DIM Malinf), and the Infect-ERA project EUGENPATH (ANR13-IFEC-0003-02) and the ANR-10-LABX-62-IBEID. PE is financed by the Fondation pour la Recherche Médicale (FRM) grant No. DEQ20120323697.

formation in the autophagic response against Salmonella. Mol Biol Cell (2011) 22:2290-300. doi:10.1091/mbc.E10-11-0893

13. Itakura E, Kishi-Itakura C, Mizushima N. The hairpin-type tail-anchored SNARE syntaxin 17 targets to autophagosomes for fusion with endosomes/ lysosomes. Cell (2012) 151:1256-69. doi:10.1016/j.cell.2012.11.001

14. Shin DM, Jeon BY, Lee HM, Jin HS, Yuk JM, Song CH, et al. Mycobacterium tuberculosis eis regulates autophagy, inflammation, and cell death through redox-dependent signaling. PLoS Pathog (2010) 6:e1001230. doi:10.1371/ journal.ppat.1001230

15. Tattoli I, Sorbara MT, Vuckovic D, Ling A, Soares F, Carneiro LAM, et al. Amino acid starvation induced by invasive bacterial pathogens triggers an innate host defense program. Cell Host Microbe (2012) 11:563-75. doi:10.1016/j.chom.2012.04.012

16. Ogawa M, Yoshimori T, Suzuki T, Sagara H, Mizushima N, Sasakawa C. Escape of intracellular Shigella from autophagy. Science (2005) 307:727-31. doi:10.1126/science.1106036

17. Mostowy S, Cossart P. Bacterial autophagy: restriction or promotion of bacterial replication? Trends Cell Biol (2012) 22:283-91. doi:10.1016/j. tcb.2012.03.006

18. Steele S, Brunton J, Kawula T. The role of autophagy in intracellular pathogen nutrient acquisition. Front Cell Infect Microbiol (2015) 5:51. doi:10.3389/ fcimb.2015.00051

19. NiuH,XiongQ, Yamamoto A,Hayashi-Nishino M, Rikihisa Y.Autophagosomes induced by a bacterial Beclin 1 binding protein facilitate obligatory intracellular infection. Proc Natl Acad Sci U S A (2012) 109:20800-7. doi:10.1073/ pnas. 1218674109

20. Niu H, Yamaguchi M, Rikihisa Y. Subversion of cellular autophagy by Anaplasma phagocytophilum. Cell Microbiol (2008) 10:593-605. doi:10.1111/j.1462-5822.2007.01068.x

21. Moreau K, Lacas-Gervais S, Fujita N, Sebbane F, Yoshimori T, Simonet $\mathrm{M}$, et al. Autophagosomes can support Yersinia pseudotuberculosis replication in macrophages. Cell Microbiol (2010) 12:1108-23. doi:10.1111/j.1462-5822.2010.01456.x

22. Pujol C, Klein KA, Romanov GA, Palmer LE, Cirota C, Zhao Z, et al. Yersinia pestis can reside in autophagosomes and avoid xenophagy in murine macrophages by preventing vacuole acidification. Infect Immun (2009) 77:2251-61. doi:10.1128/IAI.00068-09

23. Gutierrez MG, Vázquez CL, Munafó DB, Zoppino FCM, Berón W, Rabinovitch M, et al. Autophagy induction favours the generation and 
maturation of the Coxiella-replicative vacuoles. Cell Microbiol (2005) 7:981-93. doi:10.1111/j.1462-5822.2005.00527.x

24. Vázquez CL, Colombo MI. Coxiella burnetii modulates Beclin 1 and Bcl-2, preventing host cell apoptosis to generate a persistent bacterial infection. Cell Death Differ (2010) 17:421-38. doi:10.1038/cdd.2009.129

25. Berón W, Gutierrez MG, Rabinovitch M, Colombo MI. Coxiella burnetii localizes in a Rab7-labeled compartment with autophagic characteristics. Infect Immun (2002) 70:5816-21. doi:10.1128/IAI.70.10.5816-5821.2002

26. Steele S, Brunton J, Ziehr B, Taft-Benz S, Moorman N, Kawula T. Francisella tularensis harvests nutrients derived via ATG5-independent autophagy to support intracellular growth. PLoS Pathog (2013) 9:e1003562. doi:10.1371/ journal.ppat.1003562

27. Starr T, Child R, Wehrly TD, Hansen B, Hwang S, López-Otin C, et al. Selective subversion of autophagy complexes facilitates completion of the Brucella intracellular cycle. Cell Host Microbe (2012) 11:33-45. doi:10.1016/j. chom.2011.12.002

28. Mestre MB, Fader CM, Sola C, Colombo MI. Alpha-hemolysin is required for the activation of the autophagic pathway in Staphylococcus aureus-infected cells. Autophagy (2010) 6:110-25. doi:10.4161/auto.6.1.10698

29. Mestre MB, Colombo MI. cAMP and EPAC are key players in the regulation of the signal transduction pathway involved in the $\alpha$-hemolysin autophagic response. PLoS Pathog (2012) 8:e1002664. doi:10.1371/journal. ppat.1002664

30. Amer AO, Swanson MS. Autophagy is an immediate macrophage response to Legionella pneumophila. Cell Microbiol (2005) 7:765-78. doi:10.1111/j.1462-5822.2005.00509.x

31. Kagan JC, Roy CR. Legionella phagosomes intercept vesicular traffic from endoplasmic reticulum exit sites. Nat Cell Biol (2002) 4:945-54. doi:10.1038/ ncb883

32. Khweek AA, Caution K, Akhter A, Abdulrahman BA, Tazi M, Hassan H, et al. A bacterial protein promotes the recognition of the Legionella pneumophila vacuole by autophagy. Eur J Immunol (2013) 43:1333-44. doi:10.1002/ eji.201242835

33. Amer AO, Byrne BG, Swanson MS. Macrophages rapidly transfer pathogens from lipid raft vacuoles to autophagosomes. Autophagy (2005) 1:53-8. doi:10.4161/auto.1.1.1589

34. Choy A, Dancourt J, Mugo B, O'Connor TJ, Isberg RR, Melia TJ, et al. The Legionella effector RavZ inhibits host autophagy through irreversible Atg8 deconjugation. Science (2012) 338:1072-6. doi:10.1126/science.1227026

35. Rolando M, Escoll P, Nora T, Botti J, Boitez V, Bedia C, et al. Legionella pneumophila S1P-lyase targets host sphingolipid metabolism and restrains autophagy. Proc Natl Acad Sci U S A (2016) 113(7):1901-6. doi:10.1073/ pnas. 1522067113
36. Joshi AD, Swanson MS. Secrets of a successful pathogen: Legionella resistance to progression along the autophagic pathway. Front Microbiol (2011) 2:138. doi:10.3389/fmicb.2011.00138

37. Vakifahmetoglu-Norberg H, Xia H-G, Yuan J. Pharmacologic agents targeting autophagy. J Clin Invest (2015) 125:5-13. doi:10.1172/JCI73937

38. Siddiqi SH, Hawkins JE, Laszlo A. Interlaboratory drug susceptibility testing of Mycobacterium tuberculosis by a radiometric procedure and two conventional methods. J Clin Microbiol (1985) 22:919-23.

39. Stottmeier KD, Beam RE, Kubica GP. Determination of drug susceptibility of mycobacteria to pyrazinamide in 7H10 agar. Am Rev Respir Dis (1967) 96:1072-5.

40. Kim JJ, Lee HM, Shin DM, Kim W, Yuk JM, Jin HS, et al. Host cell autophagy activated by antibiotics is required for their effective antimycobacterial drug action. Cell Host Microbe (2012) 11:457-68. doi:10.1016/j.chom.2012.03.008

41. Zullo AJ, Lee S. Old antibiotics target TB with a new trick. Cell Host Microbe (2012) 11:419-20. doi:10.1016/j.chom.2012.05.002

42. Renna M, Schaffner C, Brown K, Shang S, Tamayo MH, Hegyi K, et al. Azithromycin blocks autophagy and may predispose cystic fibrosis patients to mycobacterial infection. JClin Invest (2011) 121:3554-63. doi:10.1172/ JCI46095

43. Conway KL, Kuballa P, Song J-H, Patel KK, Castoreno AB, Yilmaz OH, et al. Atg1611 is required for autophagy in intestinal epithelial cells and protection of mice from Salmonella infection. Gastroenterology (2013) 145:1347-57. doi:10.1053/j.gastro.2013.08.035

44. Kuo S-Y, Castoreno AB, Aldrich LN, Lassen KG, Goel G, Dančík V, et al. Small-molecule enhancers of autophagy modulate cellular disease phenotypes suggested by human genetics. Proc Natl Acad Sci U S A (2015) 112:E4281-7. doi:10.1073/pnas.1512289112

45. Raoult D, Houpikian P, Tissot Dupont H, Riss JM, Arditi-Djiane J, Brouqui P. Treatment of $\mathrm{Q}$ fever endocarditis: comparison of 2 regimens containing doxycycline and ofloxacin or hydroxychloroquine. Arch Intern Med (1999) 159:167-73. doi:10.1001/archinte.159.2.167

Conflict of Interest Statement: The authors declare that the research was conducted in the absence of any commercial or financial relationships that could be construed as a potential conflict of interest.

Copyright (C) 2016 Escoll, Rolando and Buchrieser. This is an open-access article distributed under the terms of the Creative Commons Attribution License (CC BY). The use, distribution or reproduction in other forums is permitted, provided the original author(s) or licensor are credited and that the original publication in this journal is cited, in accordance with accepted academic practice. No use, distribution or reproduction is permitted which does not comply with these terms. 